\title{
Does FX Volatility Affect the Distributions of Commodity Futures Returns?
}

\author{
Terrance Grieb \\ ${ }^{1}$ Department of Business, University of Idaho, Idaho, USA \\ Correspondence: Terrance Grieb, Professor of Finance, Department of Business, University of Idaho, Idaho, USA. \\ E-mail: tgrieb@uidaho.edu
}

Received: July 11, 2013

Accepted: August 5, 2013

Online Published: October 8, 2013

doi:10.5430/ijfr.v4n4p1

URL: http://dx.doi.org/10.5430/ijfr.v4n4p1

\begin{abstract}
This paper employs a two-step GARCH-M procedure to study price and volatility spillover effects from a series of foreign exchange rate returns to CME Group commodities in the grains, livestock, and energy complexes. Exchange rates are purported to have supply and demand effects on commodity prices and we hypothesize that this translates into transmissions of distributional shocks from currency returns to commodity returns distributions. The currencies are segregated into three groups: large GDP economies and major US trade partners, emerging economies, and pacific rim countries. Our results show that exchange rates for the EU, Canada, Mexico, Brazil, and Australia have the strongest and broadest transmissions of mean innovations to the observed commodities. These are all either major trade partners with the US and/or major exporters of agricultural commodities. Volatility transmissions are much less pronounced and tend to occur for lower liquidity commodities. These results have implications for models considering asset pricing, price discovery, and hedging applications for commodity returns.
\end{abstract}

Keywords: mean and volatility spillover effects, foreign exchange, commodities

\section{Introduction}

A number of studies in the finance literature examine the transmission of volatility across national boundaries. King and Wadhwani (1990), using contagion theory, argue that in a less than fully revealing equilibrium, investors and security traders respond directly to publicly available information and also observe price changes in other markets as a proxy for information that is not available in their local markets. The work done in this area has potential applications for the study of price discovery and risk-pricing interaction between asset classes.

Most of the studies in this branch of the literature employ a version of the generalized autoregressive heteroskedasticity $(\mathrm{GARCH})$ procedure in order to examine the extent to which the first and second moments of the returns distributions of one market are influenced by information shocks in other markets. Koutmos and Booth (1995) and Kanas (1998) use a two-stage bivariate Exponential GARCH (EGARCH) model to observe volatility spillovers between developed countries. These studies document larger conditional volatility in down-markets than in up-markets, consistent with the leverage effect (Black 1976). In addition, both studies find significantly higher degrees of volatility transmission among all markets after the October 1987 stock market crash. Similar transmissions for markets in developed countries are documented by Theodossiou and Lee (1993), and by Theodossiou, Kahya, Koutmos, and Christofi (1997).

A number of other studies also use a two-step GARCH-M procedure which allows them to simultaneously observe transmission effects for a large number of countries. Hamao, Masulius, and $\mathrm{Ng}$ (1990) use this procedure for price and volatility spillovers pre- and post-October 1987 and find limited volatility spillovers pre-crash, with strong price and volatility spillovers after the crash. Liu and Pan (1997) apply this procedure to emerging markets and obtain similar results. Conrad, Gultiken, and Kaul (1991) use the two-step procedure to observe small-cap vs. large-cap stocks and find that volatility shocks to large firm returns are transmitted to small firms.

While this branch of the literature deals mostly with international investments, this paper suggests that an interesting application of King and Wadhwani's contagion theory, and of the mean-return and volatility transmission methodology, can be applied to commodity markets within the United States. 
In particular, commodity markets are quickly becoming a more standard asset class within domestic US markets, so asset pricing models and price discovery behavior have become topics of interest. For example, Driesprong, Jacobsen, and Maat (2008) use a standard OLS regression model to document a relationship between oil futures and world stock market returns. Lien and Yang (2008) demonstrate that the minimum variance hedge ratio for a given commodity is affected differently by a positive basis (i.e., backwardation) than a negative basis (i.e., contango).

Trolle and Schwartz (2009) demonstrate that volatility for the derivatives of a given commodity may have both spanned and unspanned components, and that both types of volatility must be accounted for when estimating the instantaneous volatility for option contracts and futures curve dynamics. Likewise, Duan and Pliska (2004) observe the S\&P500 and NASDAQ 100 and show that when stochastic volatility is present, option price estimations must reflect co-integration dynamics across assets. Nakajima and Ohashi (2012) obtain similar results for WTI crude and heating oil derivatives.

Inter-commodity information transfer has also become an important topic. Tse (1998) applies an EGARCH model to Euroyen and Eurodollar futures and finds no evidence of volatility spillovers, although the results are consistent with covered interest arbitrage. Chng (2009) uses a vector autoregression to document information transfer across auto industry related commodities (i.e., rubber, palladium and gasoline). Badshah, Frijns, and Tourani-Rad (2013) use a multivariate GARCH to observe spillovers between the volatility indices for stock (i.e., the VIX), gold (i.e., GVZ), and the Euro-Dollar exchange rate (i.e., EVZ) and find spillover from the VIX to the other indices. Cortazar and Severino (2008) use a Kalman filter procedure to create an inter-commodity pricing model for WTI and Brent crude based on common and commodity specific factors. However, a broader examination of information transfer effects across commodities is not currently represented in the literature.

Our study contributes to this literature by observing information spill-over to commodity markets from the foreign exchange markets. We employ the two-stage GARCH-M procedure proposed by Hamao et al (1990) to study mean and volatility spillover effects to the futures contracts of nine commodities from a series of foreign exchange spot rates. Commodity markets play a large role in the balance of trade for the US, and changes in exchange rates are widely believed to have supply and demand effects on the prices of those commodities. If distributional shocks to the forex market are transmitted to the returns distributions of commodities traded in the US, then this has important implications for asset pricing, price discovery, and hedging models.

The format of this paper is as follows. Section 2 describes the data and methodology. Section 3 presents the empirical results and the conclusions are summarized in Section 4.

\section{Data and Methodology}

\subsection{Data}

The data used in this study consists of daily returns for futures contracts on nine different commodities traded on CME Group exchanges and for spot rates on eight major currencies relative to the US dollar. (Note 1) The data spans the period from January 1, 2001 to December 31, 2011. The data was obtained from Bloomberg and we use the daily prices for the rolling front month to estimate returns for the futures contracts. The front month generally refers to the month closest to maturity and each rolling front month series uses the contract with the highest volume and open interest as the front month. Outstanding contracts that have "rolled" are characterized by decreased liquidity due to shrinking volume and open interest and by decreased price sensitivity to market dynamics.

Futures contracts for physical commodities generally provide higher liquidity and better price discovery than the spot market because they provide a single price for the underlying. In contrast, commodity spot markets provide a range of price quotes depending on the location of delivery and the quality of the deliverable. As a result, futures contracts are widely used as the primary tool for hedging and speculation for a given commodity.

We divide the nine different commodities into three complexes. The first four commodities are corn, rough rice, soybeans, and wheat, which are the major commodities traded in the grains complex on the Chicago Board of Trade. Each of the contracts has significant trading volume (although rice is the least liquid of the four) and each has corresponding options contracts that trade with the futures contract as the underlying.

The next three commodities are feeder cattle, lean hogs, and live cattle, which are the major commodities traded in the livestock complex on the Chicago Mercantile Exchange. Each of these futures contracts has a corresponding options contract and trading in these commodities occurs continuously on the electronic Globex platform. This complex tends to have lower volume than the major grains commodities, but they have higher liquidity than the other CME agricultural complexes (i.e., dairy, lumber, and the softs). 
The last two commodities are light sweet crude (West Texas Intermediate) and Henry Hub natural gas. Both are traded on the NYMEX and they are, respectively, the first and second largest volume futures contracts on a physical commodity. Light sweet crude oil is the world's primary forum for crude oil trading, and is a globally dollar-denominated asset. The Henry Hub natural gas contract represents the national benchmark for the price of natural gas, which accounts for almost a quarter of the energy usage in the United States.

The eight currencies are allocated across three different groups. (Note 2) The first currency group represents large GDP countries and major US trade partners. This group contains the European Union, Japan, United Kingdom, Canada, and Mexico. (Note 3) The second group represents emerging markets of interest and includes the currencies of Brazil, Mexico, and South Korea. Each of these countries has a well established market for their currencies and significant import/export profiles for agricultural commodities. (Note 4) The third group represents the major Asian (aka Pacific Rim) countries and includes the currencies of Australia, Japan, New Zealand, and South Korea. (Note 5)

Table 1 provides summary statistics for the daily returns for each of the currency exchange rates and for each of the commodities. The skewness and kurtosis measures for all the observed commodities are quite high, indicating that none of the daily return series are normally distributed. The Jarque-Bera statistics reject normality in all cases at the one-percent level of significance.

Table 1. Summary statistics

Panel A. Currency returns

\begin{tabular}{|c|c|c|c|c|c|c|c|c|c|}
\hline & Australia & Brazil & Canada & Euro Zone & $\begin{array}{c}\text { United } \\
\text { Kingdom }\end{array}$ & Japan & $\begin{array}{l}\text { South } \\
\text { Korea }\end{array}$ & Mexico & $\begin{array}{c}\text { New } \\
\text { Zealand }\end{array}$ \\
\hline Mean & -0.0002 & 0.0000 & -0.0001 & -0.0001 & 0.0000 & -0.0001 & 0.0001 & -0.0001 & -0.0002 \\
\hline Median & -0.0007 & -0.0003 & -0.0003 & -0.0002 & -0.0001 & -0.0001 & -0.0002 & -0.0003 & -0.0007 \\
\hline Maximum & 0.0729 & 0.0640 & 0.0325 & 0.0277 & 0.0562 & 0.0550 & 0.0703 & 0.0487 & 0.0729 \\
\hline Minimum & -0.0828 & -0.1034 & -0.0400 & -0.0347 & -0.0293 & -0.0350 & -0.0665 & -0.0497 & -0.0828 \\
\hline Std. Dev. & 0.0093 & 0.0116 & 0.0064 & 0.0067 & 0.0061 & 0.0067 & 0.0071 & 0.0083 & 0.0093 \\
\hline Skewness & 0.4453 & 0.1624 & 0.1974 & 0.0613 & 0.5335 & 0.0501 & 0.8108 & 0.1731 & 0.4453 \\
\hline Kurtosis & 11.8843 & 9.5715 & 5.5335 & 4.3033 & 7.1871 & 6.7085 & 16.8469 & 5.4718 & 11.8843 \\
\hline \multirow[t]{2}{*}{ Jarque-Bera } & 9178.26 & 4983.77 & 756.89 & 197.26 & 2149.41 & 1584.48 & 22376.4 & 717.16 & 9178.26 \\
\hline & $* * *$ & $* * *$ & $* * *$ & $* * *$ & $* * *$ & $* * *$ & $* * *$ & $* * *$ & $* * *$ \\
\hline Observations & 2,763 & 2,763 & 2,763 & 2,763 & 2,763 & 2,763 & 2,763 & 2,763 & 2,763 \\
\hline \multicolumn{10}{|c|}{ Panel B. Commodity returns } \\
\hline & Corn & $\begin{array}{l}\text { Rough } \\
\text { Rice }\end{array}$ & Soybeans & Wheat & $\begin{array}{l}\text { Feeder } \\
\text { Cattle }\end{array}$ & Hogs & Live Cattle & $\begin{array}{c}\text { WTI } \\
\text { Crude }\end{array}$ & $\begin{array}{c}\text { Natural } \\
\text { Gas }\end{array}$ \\
\hline Mean & 0.0004 & 0.0003 & 0.0003 & 0.0003 & 0.0002 & 0.0002 & 0.0002 & 0.0005 & -0.0003 \\
\hline Median & 0.0000 & 0.0000 & 0.0008 & -0.0005 & 0.0003 & 0.0000 & 0.0000 & 0.0008 & -0.0009 \\
\hline Maximum & 0.1241 & 0.0857 & 0.0653 & 0.1108 & 0.0431 & 0.1332 & 0.0764 & 0.1278 & 0.2344 \\
\hline Minimum & -0.0812 & -0.1648 & -0.1650 & -0.0976 & -0.0615 & -0.2123 & -0.0987 & -0.1572 & -0.2142 \\
\hline Std. Dev. & 0.0185 & 0.0172 & 0.0168 & 0.0206 & 0.0095 & 0.0188 & 0.0109 & 0.0231 & 0.0335 \\
\hline Skewness & 0.1796 & -0.3126 & -0.7218 & 0.1241 & -0.3815 & -0.1446 & -0.1422 & -0.2772 & 0.5436 \\
\hline Kurtosis & 5.2848 & 8.3679 & 8.6497 & 4.9155 & 6.1441 & 18.9655 & 11.1337 & 6.0006 & 7.3732 \\
\hline \multirow[t]{2}{*}{ Jarque-Bera } & 615.84 & 3362.28 & $3914.6 * * *$ & $429.49 * * *$ & $1205.1 * * *$ & 29354.6 & 7625.65 & 1071.91 & 2337.87 \\
\hline & $* * *$ & $* * *$ & & & & $* * *$ & $* * *$ & $* * *$ & $* * *$ \\
\hline Observations & 2,763 & 2,763 & 2,763 & 2,763 & 2,763 & 2,763 & 2,763 & 2,763 & 2,763 \\
\hline
\end{tabular}

** Significant at the $5 \%$ level. $\quad * * *$ Significant at the $1 \%$ level. 


\subsection{Methodology}

We use a two-stage ARMA(1,1)-GARCH(1,1)-M procedure to capture both mean return and volatility transmissions. Consistent with other studies that have employed a two-stage GARCH procedure, the autoregressive term corrects for serial correlation in the returns.

Let $i$ represent the returns series for each of the eight exchange rates and for each of the nine commodity futures. In the first stage we estimate the following univariate model.

$$
\begin{gathered}
R_{i, t}=\beta_{i, 0}+\beta_{i, 1} R_{i, t-1}+\beta_{i, 2} \varepsilon_{i, t-1}+\beta_{i, 3} \sigma_{i, t}^{2}+\varepsilon_{i, t} \\
\sigma_{i, t}^{2}=\gamma_{i, 0}+\gamma_{i, 1} \varepsilon_{t-1}^{2}+\gamma_{i, 2} \sigma_{t-1}^{2}
\end{gathered}
$$

where $R_{i, t}$ is the return for exchange rate or commodity $i$, and $\sigma_{i, t}^{2}$ is the time-varying conditional variance of the error term $\varepsilon_{i, t}$. The estimated variance in each period is sensitive to inordinately large positive or negative returns in the previous period. This allows the model to account for volatility clustering and serial correlation in the return series. In addition, the coefficients $\gamma_{i, 1}$ and $\gamma_{i, 2}$ in the conditional volatility equation test the null hypothesis of no conditional variance effects in the time series. Due to the non-normality of the returns data we estimate quasi-maximum likelihood covariances and standard errors as described by Bollerslev and Wooldridge (1992). We use the Berndt-Hall-Hall-Hausman iteration process to obtain these estimates.

In the second stage of the analysis, we follow Koutmos and Booth (1995) and use the standardized residuals and their squares estimated from stage one as follows:

$$
\begin{gathered}
R_{i, t}=\delta_{i, 0}+\delta_{i, 1} R_{i, t-1}+\delta_{i, 2} \varepsilon_{i, t-1}+\delta_{i, 3} \sigma_{i, t}^{2}+\sum_{j=1}^{n} \delta_{i, 3+j} \hat{\eta}_{j, t}+\varepsilon_{i, t} \\
\sigma_{i, t}^{2}=\lambda_{i, 0}+\lambda_{i, 2} \varepsilon_{t-1}^{2}+\lambda_{i, 3} \sigma_{t-1}^{2}+\sum_{j=1}^{n} \lambda_{i, 3+j} \widehat{\eta}_{j, t}^{2}
\end{gathered}
$$

where $\hat{\eta}_{j}$ represents the standardized residuals $\left(=\widehat{\varepsilon}_{j} / \widehat{\sigma}_{j}\right)$ estimated from stage one, and $j$ indicates the exchange rate transmitting mean and volatility effects to commodity $i$. In the case of the first group of exchange rates (i.e., large GDP economies and major US trade partners), $j=1$ to 5 and corresponds to standardized residuals for currency exchange rates for the EU, Japan, UK, Canada, and Mexico, respectively. Likewise, for the second group of currencies, $j=1$ to 3 and corresponds to Brazil, Mexico, and South Korea. Finally, for the third group of currencies, $j$ $=1$ to 4 and corresponds to Australia, Japan, New Zealand, and South Korea. Accordingly, the coefficients $\delta_{i, 3+j}$ and $\gamma_{i, 3+j}$ test the null hypotheses of no mean spillover effect and no volatility spillover effect, respectively, from currency $j$ to the observed commodity.

\section{Results}

\subsection{Univariate Regressions}

We estimate an $\operatorname{ARMA}(1,1)-\operatorname{GARCH}(1,1)-\mathrm{M}$ model using equations (1) and (2) and generate a time series of standardized residuals and squared standardized residuals for each of the currencies and commodities in the sample. The results from the univariate GARCH estimations for the observed currencies and commodities are reported in Tables 2 and 3, respectively. The ARCH-LM statistic that tests for ARCH effects in the standardized residuals is uniformly insignificant. This suggests that the ARMA(1,1)-GARCH(1,1)-M specification fits the data very well and that the resulting standardized residuals are well-specified for use in the stage 2 regressions. Likewise, the ARCH and GARCH coefficients in the conditional volatility equations are uniformly significant at the $1 \%$ level for every series, indicating the need to account for conditional volatility when modeling the time series. 
Table 2. Univariate ARMA-GARCH-M results for currency returns

\begin{tabular}{|c|c|c|c|c|c|c|c|c|c|}
\hline & Australia & Brazil & Canada & $\begin{array}{l}\text { Euro } \\
\text { Zone }\end{array}$ & $\begin{array}{c}\text { Great } \\
\text { Britain }\end{array}$ & Japan & $\begin{array}{l}\text { South } \\
\text { Korea }\end{array}$ & Mexico & $\begin{array}{c}\text { New } \\
\text { Zealand }\end{array}$ \\
\hline $\begin{array}{l}\beta_{i, 0} \\
(\mathrm{x} 1,000)\end{array}$ & $\begin{array}{c}0.278 \\
(0.207)\end{array}$ & $\begin{array}{c}-0.385 \\
\left(0.185^{* *}\right)\end{array}$ & $\begin{array}{l}-0.172 \\
(0.149)\end{array}$ & $\begin{array}{c}-0.533 \\
(0.228 * *)\end{array}$ & $\begin{array}{l}-0.369 \\
(0.211)\end{array}$ & $\begin{array}{l}-0.413 \\
(0.357)\end{array}$ & $\begin{array}{l}-0.089 \\
(0.119)\end{array}$ & $\begin{array}{l}-0.065 \\
(0.126)\end{array}$ & $\begin{array}{c}0.605 \\
\left(0.31^{* *}\right)\end{array}$ \\
\hline$\beta_{i, 1}$ & $\begin{array}{c}0.588 \\
(0.644)\end{array}$ & $\begin{array}{c}0.460 \\
(0.306)\end{array}$ & $\begin{array}{c}0.599 \\
(0.310)\end{array}$ & $\begin{array}{l}-0.384 \\
(0.354)\end{array}$ & $\begin{array}{l}-0.275 \\
(0.346)\end{array}$ & $\begin{array}{c}-0.658 \\
\left(0.307^{* *}\right)\end{array}$ & $\begin{array}{c}0.008 \\
(0.479)\end{array}$ & $\begin{array}{l}-0.403 \\
(0.214)\end{array}$ & $\begin{array}{l}-0.307 \\
(0.605)\end{array}$ \\
\hline$\beta_{i, 2}$ & $\begin{array}{l}-0.605 \\
(0.633)\end{array}$ & $\begin{array}{l}-0.502 \\
(0.300)\end{array}$ & $\begin{array}{c}-0.630 \\
\left(0.301^{* *}\right)\end{array}$ & $\begin{array}{c}0.369 \\
(0.356)\end{array}$ & $\begin{array}{c}0.276 \\
(0.347)\end{array}$ & $\begin{array}{c}0.660 \\
(0.307 * *)\end{array}$ & $\begin{array}{l}-0.050 \\
(0.478)\end{array}$ & $\begin{array}{c}0.350 \\
(0.219)\end{array}$ & $\begin{array}{c}0.301 \\
(0.607)\end{array}$ \\
\hline$\beta_{i, 3}$ & $\begin{array}{c}1.611 \\
(3.147)\end{array}$ & $\begin{array}{l}-0.139 \\
(2.042)\end{array}$ & $\begin{array}{c}0.312 \\
(4.593)\end{array}$ & $\begin{array}{c}8.596 \\
(5.624)\end{array}$ & $\begin{array}{c}8.775 \\
(6.497)\end{array}$ & $\begin{array}{c}9.937 \\
(8.858)\end{array}$ & $\begin{array}{l}-2.813 \\
(3.502)\end{array}$ & $\begin{array}{c}0.347 \\
(3.673)\end{array}$ & $\begin{array}{l}-4.127 \\
(4.300)\end{array}$ \\
\hline $\begin{array}{l}\gamma_{i, 0} \\
(\mathrm{x} 100,000)\end{array}$ & $\begin{array}{c}0.077 \\
(0.03 * * *)\end{array}$ & $\begin{array}{c}0.166 \\
(0.04 * * *)\end{array}$ & $\begin{array}{c}0.018 \\
(0.011)\end{array}$ & $\begin{array}{c}0.016 \\
(0.016)\end{array}$ & $\begin{array}{c}0.024 \\
(0.024)\end{array}$ & $\begin{array}{c}0.131 \\
(0.089)\end{array}$ & $\begin{array}{c}0.045 \\
(0.02 * * *)\end{array}$ & $\begin{array}{c}0.045 \\
(0.01 * * *)\end{array}$ & $\begin{array}{c}0.119 \\
(0.04 * * *)\end{array}$ \\
\hline$\gamma_{i, 1}$ & $\begin{array}{c}0.070 \\
(0.01 * * *)\end{array}$ & $\begin{array}{c}0.135 \\
(0.02 * * *)\end{array}$ & $\begin{array}{c}0.052 \\
\left(0.01^{* * *}\right)\end{array}$ & $\begin{array}{c}0.034 \\
(0.01 * * *)\end{array}$ & $\begin{array}{c}0.037 \\
\left(0.01^{* * *}\right)\end{array}$ & $\begin{array}{c}0.049 \\
(0.01 * * *)\end{array}$ & $\begin{array}{c}0.082 \\
(0.02 * * *)\end{array}$ & $\begin{array}{c}0.086 \\
(0.02 * * *)\end{array}$ & $\begin{array}{c}0.058 \\
(0.01 * * *)\end{array}$ \\
\hline$\gamma_{i, 2}$ & $\begin{array}{c}0.920 \\
(0.01 * * *)\end{array}$ & $\begin{array}{c}0.857 \\
(0.02 * * *)\end{array}$ & $\begin{array}{c}0.944 \\
(0.01 * * *)\end{array}$ & $\begin{array}{c}0.963 \\
(0.01 * * *)\end{array}$ & $\begin{array}{c}0.956 \\
\left(0.01^{* * *}\right)\end{array}$ & $\begin{array}{c}0.919 \\
(0.02 * * *)\end{array}$ & $\begin{array}{c}0.908 \\
\left(0.01^{* * *}\right)\end{array}$ & $\begin{array}{c}0.905 \\
(0.01 * * *)\end{array}$ & $\begin{array}{c}0.926 \\
(0.01 * * *)\end{array}$ \\
\hline ARCH-LM & 0.2932 & 0.1834 & 3.7530 & 2.8220 & 0.2121 & 0.1961 & 0.0890 & 0.0470 & 0.2760 \\
\hline
\end{tabular}

NOTES: Numbers in parenthesis are Bollerslev-Wooldridge robust standard errors. ARCH-LM is an F-test for $\mathrm{ARCH}$ effects in the standardized residuals.

** Significant at the $5 \%$ level. $\quad * * *$ Significant at the $1 \%$ level.

Table 3. Univariate ARMA-GARCH-M results for commodity returns

\begin{tabular}{|c|c|c|c|c|c|c|c|c|c|}
\hline & Corn & Rice & Soybeans & Wheat & $\begin{array}{c}\text { Feeder } \\
\text { Cattle }\end{array}$ & Hogs & Live Cattle & Crude & $\begin{array}{c}\text { Natural } \\
\text { Gas }\end{array}$ \\
\hline $\begin{array}{l}\beta_{i, 0} \\
(\mathrm{x} 1,000)\end{array}$ & $\begin{array}{c}-0.06100 \\
(0.569)\end{array}$ & $\begin{array}{c}1.738 \\
\left(0.857^{* *}\right)\end{array}$ & $\begin{array}{c}1.714 \\
(0.54 * * *)\end{array}$ & $\begin{array}{c}1.141 \\
(0.750)\end{array}$ & $\begin{array}{l}-0.439 \\
(0.423)\end{array}$ & $\begin{array}{c}0.755 \\
(6.870)\end{array}$ & $\begin{array}{c}1.13 \\
(1.199)\end{array}$ & $\begin{array}{c}0.922 \\
(0.915)\end{array}$ & $\begin{array}{c}1.609 \\
((1.384)\end{array}$ \\
\hline$\beta_{i, 1}$ & $\begin{array}{l}-0.14446 \\
(0.4644)\end{array}$ & $\begin{array}{l}-0.2630 \\
(0.2450)\end{array}$ & $\begin{array}{c}0.0480 \\
(3.2765)\end{array}$ & $\begin{array}{c}0.0773 \\
(0.0801)\end{array}$ & $\begin{array}{c}0.1862 \\
(0.2202)\end{array}$ & $\begin{array}{c}0.9908 \\
\left(0.01^{* * *}\right)\end{array}$ & $\begin{array}{c}0.4630 \\
(0.5879)\end{array}$ & $\begin{array}{l}-0.4960 \\
(0.8081)\end{array}$ & $\begin{array}{l}-0.5089 \\
(0.3492)\end{array}$ \\
\hline$\beta_{i, 2}$ & $\begin{array}{l}0.18252 \\
(0.4604)\end{array}$ & $\begin{array}{l}0.322573 \\
(0.2403)\end{array}$ & $\begin{array}{l}-0.0532 \\
(3.2737)\end{array}$ & $\begin{array}{l}-0.0873 \\
(0.0827)\end{array}$ & $\begin{array}{l}-0.1113 \\
(0.2224)\end{array}$ & $\begin{array}{c}-0.9972 \\
(0.01 * * *)\end{array}$ & $\begin{array}{l}-0.4616 \\
(0.5881)\end{array}$ & $\begin{array}{c}0.4818 \\
(0.8155)\end{array}$ & $\begin{array}{c}0.4745 \\
(0.3573)\end{array}$ \\
\hline$\beta_{i, 3}$ & $\begin{array}{l}1.46328 \\
(1.8688)\end{array}$ & $\begin{array}{l}-4.75933 \\
(3.0033)\end{array}$ & $\begin{array}{c}-5.7351 \\
\left(2.18^{* * *}\right)\end{array}$ & $\begin{array}{l}-2.6283 \\
(2.0074)\end{array}$ & $\begin{array}{c}8.5331 \\
(5.2268)\end{array}$ & $\begin{array}{c}-1.8150 \\
(19.2855)\end{array}$ & $\begin{array}{l}-7.8182 \\
(10.128)\end{array}$ & $\begin{array}{c}0.0634 \\
(1.9841)\end{array}$ & $\begin{array}{l}-1.6231 \\
(1.3829)\end{array}$ \\
\hline $\begin{array}{l}\gamma_{i, 0} \\
(\mathrm{x} 10,000)\end{array}$ & $\begin{array}{c}0.0293 \\
(0.014 * *)\end{array}$ & $\begin{array}{c}0.0250 \\
(0.01 * * *)\end{array}$ & $\begin{array}{c}0.0310 \\
\left(0.014^{* *}\right)\end{array}$ & $\begin{array}{c}0.0310 \\
(0.01 * * *)\end{array}$ & $\begin{array}{c}0.0087 \\
(0.0087)\end{array}$ & $\begin{array}{c}0.3730 \\
(0.5120)\end{array}$ & $\begin{array}{c}0.1670 \\
(0.1090)\end{array}$ & $\begin{array}{c}0.0974 \\
\left(0.038^{* *}\right)\end{array}$ & $\begin{array}{c}0.1450 \\
(0.0582 * *)\end{array}$ \\
\hline$\gamma_{i, 1}$ & $\begin{array}{c}0.05134 \\
(0.01 * * *)\end{array}$ & $\begin{array}{l}0.029231 \\
(0.01 * * *)\end{array}$ & $\begin{array}{l}0.048766 \\
\left(0.01^{* * *}\right)\end{array}$ & $\begin{array}{c}0.0337 \\
\left(0.01^{* * *}\right)\end{array}$ & $\begin{array}{c}0.0321 \\
\left(0.01^{* * *}\right)\end{array}$ & $\begin{array}{c}0.0023 \\
(0.0052)\end{array}$ & $\begin{array}{c}0.0320 \\
(0.0192)\end{array}$ & $\begin{array}{c}0.0523 \\
(0.02 * * *)\end{array}$ & $\begin{array}{c}0.0476 \\
(0.009 * * *)\end{array}$ \\
\hline$\gamma_{i, 2}$ & $\begin{array}{c}0.9414 \\
(0.01 * * *)\end{array}$ & $\begin{array}{c}0.9629 \\
\left(0.011^{* * *}\right)\end{array}$ & $\begin{array}{c}0.9405 \\
(0.01 * * *)\end{array}$ & $\begin{array}{c}0.9594 \\
\left(0.01^{* * *}\right)\end{array}$ & $\begin{array}{c}0.9584 \\
\left(0.01^{* * *}\right)\end{array}$ & $\begin{array}{c}0.8930 \\
\left(0.15^{* * *}\right)\end{array}$ & $\begin{array}{c}0.8281 \\
\left(0.10^{* * *}\right)\end{array}$ & $\begin{array}{c}0.9277 \\
(0.019 * * *)\end{array}$ & $\begin{array}{c}0.9400 \\
(0.012 * * *)\end{array}$ \\
\hline ARCH-LM & 0.2138 & 0.5178 & 0.4956 & 0.1425 & 0.5086 & 0.0370 & 0.2097 & 0.0607 & 0.2561 \\
\hline
\end{tabular}

NOTES: Numbers in parenthesis are Bollerslev-Wooldridge robust standard errors. ARCH-LM is an F-test for $\mathrm{ARCH}$ effects in the standardized residuals.

** Significant at the $5 \%$ level. $\quad * * *$ Significant at the $1 \%$ level. 


\subsection{Mean and Volatility Spillovers from Currencies to Commodities}

Table 4 shows the results for the mean-return and volatility transmissions to each of the commodities from the exchange rate returns for large GDP economies and major US trade partners as estimated by equations 3 and 4 . The $\delta$ coefficients test the null hypothesis of price spillovers resulting from innovations in the mean of the exchange rate returns distribution transmitting effects to the mean of each commodity's returns distribution. The price spillover effect is widely distributed across the commodities for the Euro, the Canadian dollar, and the Mexican peso. Every commodity except natural gas receives from at least two of these currencies, and each of the currencies affects at least six of the nine commodities. Most notable is the Canadian dollar where innovations in the mean of its distribution is transmitted to eight of the nine commodities - including natural gas - with the sole exception being feeder cattle. Both Japan and the UK exhibit much lower price spillover effects, with the Japanese yen affecting only feeder cattle and crude and the UK pound affecting only soybeans.

In similar fashion, the $\gamma$ coefficients test the null hypothesis of spillovers from innovations in the variance of the exchange rate returns distribution to the variance of the commodity returns distribution. The volatility transmission effects are much less pronounced with only four significant transmissions across the entire data sample. However, we note that two of those volatility spillover cases involved hogs, which receives volatility transmissions from Japan and Canada.

Table 4. Mean and volatility spillovers from large economies and major US trade partners

\begin{tabular}{|c|c|c|c|c|c|c|c|c|c|}
\hline & Corn & Rice & Soybeans & Wheat & F. Cattle & Hogs & L. Cattle & Crude & Nat. Gas \\
\hline$\delta_{i, 3}$ & $\begin{array}{c}1.639 \\
(1.919)\end{array}$ & $\begin{array}{c}1.040 \\
(3.131)\end{array}$ & $\begin{array}{c}-6.735 \\
(1.92 * * *)\end{array}$ & $\begin{array}{c}-5.733 \\
(2.09 * * *)\end{array}$ & $\begin{array}{c}8.799 \\
(5.185)\end{array}$ & $\begin{array}{l}-0.132 \\
(0.105)\end{array}$ & $\begin{array}{c}-17.005 \\
\left(8.115^{* *}\right)\end{array}$ & $\begin{array}{c}-0.534 \\
(1.906)\end{array}$ & $\begin{array}{l}-2.806 \\
(2.405)\end{array}$ \\
\hline $\begin{array}{l}\delta_{i, \text { Euro }} \\
(\mathrm{x} 1,000)\end{array}$ & $\begin{array}{c}-1.475 \\
\left(0.45^{* * *}\right)\end{array}$ & $\begin{array}{c}-1.002 \\
(0.465 * *)\end{array}$ & $\begin{array}{l}-0.429 \\
(0.271)\end{array}$ & $\begin{array}{c}-1.426 \\
(0.53 * * *)\end{array}$ & $\begin{array}{c}0.498 \\
\left(0.237^{* *}\right)\end{array}$ & $\begin{array}{c}1.672 \\
(0.58 * * *)\end{array}$ & $\begin{array}{l}-0.062 \\
(0.309)\end{array}$ & $\begin{array}{c}-2.787 \\
(0.60 * *)\end{array}$ & $\begin{array}{l}-1.462 \\
(1.023)\end{array}$ \\
\hline $\begin{array}{l}\delta_{i, \mathrm{Japan}} \\
(\mathrm{x} 1,000)\end{array}$ & $\begin{array}{c}0.360 \\
(0.339)\end{array}$ & $\begin{array}{c}0.406 \\
(0.339)\end{array}$ & $\begin{array}{c}0.338 \\
(0.342)\end{array}$ & $\begin{array}{c}0.540 \\
(0.391)\end{array}$ & $\begin{array}{c}0.376 \\
(0.168 * *)\end{array}$ & $\begin{array}{c}0.153 \\
(0.320)\end{array}$ & $\begin{array}{c}0.392 \\
(0.205)\end{array}$ & $\begin{array}{c}1.697 \\
\left(0.43^{* * *}\right)\end{array}$ & $\begin{array}{l}-0.237 \\
(0.682)\end{array}$ \\
\hline $\begin{array}{l}\delta_{i, \mathrm{UK}} \\
(\mathrm{x} 1,000)\end{array}$ & $\begin{array}{l}-0.250 \\
(0.439)\end{array}$ & $\begin{array}{l}-0.656 \\
(0.417)\end{array}$ & $\begin{array}{c}-0.997 \\
(0.38 * * *)\end{array}$ & $\begin{array}{l}-0.439 \\
(0.491)\end{array}$ & $\begin{array}{l}-0.357 \\
(0.228)\end{array}$ & $\begin{array}{l}-0.796 \\
(0.598)\end{array}$ & $\begin{array}{l}-0.395 \\
(0.276)\end{array}$ & $\begin{array}{l}-0.468 \\
(0.549)\end{array}$ & $\begin{array}{l}-0.722 \\
(0.825)\end{array}$ \\
\hline $\begin{array}{l}\delta_{i, \text { Canada }} \\
(\mathrm{x} 1,000)\end{array}$ & $\begin{array}{c}-0.808 \\
\left(0.35^{* * *}\right)\end{array}$ & $\begin{array}{c}-0.770 \\
(0.370 * *)\end{array}$ & $\begin{array}{c}-2.335 \\
\left(0.36^{* * *}\right)\end{array}$ & $\begin{array}{c}-1.159 \\
(0.34 * * *)\end{array}$ & $\begin{array}{l}-0.349 \\
(0.193)\end{array}$ & $\begin{array}{c}-1.551 \\
(0.50 * * *)\end{array}$ & $\begin{array}{c}-0.729 \\
(0.27 * * *)\end{array}$ & $\begin{array}{c}-4.592 \\
(0.43 * * *)\end{array}$ & $\begin{array}{c}-2.763 \\
(0.90 * * *)\end{array}$ \\
\hline $\begin{array}{l}\delta_{i, \text { Mexico }} \\
(\mathrm{x} 1,000)\end{array}$ & $\begin{array}{c}-1.471 \\
(0.31 * * *)\end{array}$ & $\begin{array}{l}-0.619 \\
(0.320)\end{array}$ & $\begin{array}{c}-0.960 \\
(0.31 * * *)\end{array}$ & $\begin{array}{c}-0.837 \\
(0.360 * *)\end{array}$ & $\begin{array}{c}-0.496 \\
(0.18 * * *)\end{array}$ & $\begin{array}{c}0.196 \\
(0.392)\end{array}$ & $\begin{array}{c}-0.557 \\
(0.23 * * *)\end{array}$ & $\begin{array}{c}-1.529 \\
(0.41 * * *)\end{array}$ & $\begin{array}{l}-1.316 \\
(0.797)\end{array}$ \\
\hline$\gamma_{i, 2}$ & $\begin{array}{c}0.050 \\
(0.01 * * *)\end{array}$ & $\begin{array}{c}0.030 \\
(0.011)\end{array}$ & $\begin{array}{c}0.179 \\
(0.03 * * *)\end{array}$ & $\begin{array}{c}0.027 \\
(0.01 * * *)\end{array}$ & $\begin{array}{c}0.031 \\
\left(0.01^{* *}\right)\end{array}$ & $\begin{array}{c}0.165 \\
\left(0.03^{* * *}\right)\end{array}$ & $\begin{array}{c}0.038 \\
(0.021)\end{array}$ & $\begin{array}{c}0.056 \\
(0.01 * * *)\end{array}$ & $\begin{array}{c}0.062 \\
(0.01 * * *)\end{array}$ \\
\hline$\gamma_{i, 3}$ & $\begin{array}{c}0.940 \\
(0.01 * * *)\end{array}$ & $\begin{array}{c}0.960 \\
(0.011)\end{array}$ & $\begin{array}{c}0.736 \\
(0.02 * * *)\end{array}$ & $\begin{array}{c}0.967 \\
(.01 * * *)\end{array}$ & $\begin{array}{c}0.960 \\
\left(0.01^{* * *}\right)\end{array}$ & $\begin{array}{c}0.555(0.0 \\
0.02 * * *)\end{array}$ & $\begin{array}{c}0.562 \\
\left(0.11^{* * *}\right)\end{array}$ & $\begin{array}{c}0.920 \\
(0.02 * * *)\end{array}$ & $\begin{array}{c}0.906 \\
(0.02 * * *)\end{array}$ \\
\hline $\begin{array}{l}\gamma_{i, \text { Euro }} \\
(100,000)\end{array}$ & $\begin{array}{c}0.023 \\
(0.301)\end{array}$ & $\begin{array}{c}0.068 \\
(0.237)\end{array}$ & $\begin{array}{c}-1.680 \\
\left(0.56^{* * *}\right)\end{array}$ & $\begin{array}{c}0.039 \\
(0.190)\end{array}$ & $\begin{array}{c}0.085 \\
(0.045)\end{array}$ & $\begin{array}{c}3.310 \\
(1.750)\end{array}$ & $\begin{array}{l}-0.148 \\
(0.255)\end{array}$ & $\begin{array}{c}0.693 \\
(0.623)\end{array}$ & $\begin{array}{l}-3.000 \\
(1.710)\end{array}$ \\
\hline $\begin{array}{l}\gamma_{\mathrm{i}, \mathrm{Japan}} \\
(\mathrm{x} 100,000)\end{array}$ & $\begin{array}{c}0.245 \\
(0.179)\end{array}$ & $\begin{array}{c}0.262 \\
(0.165)\end{array}$ & $\begin{array}{c}0.087 \\
(0.182)\end{array}$ & $\begin{array}{c}0.083 \\
(0.129)\end{array}$ & $\begin{array}{l}-0.041 \\
(0.038)\end{array}$ & $\begin{array}{c}-2.140 \\
(0.41 * * *)\end{array}$ & $\begin{array}{l}-0.214 \\
(0.175)\end{array}$ & $\begin{array}{l}-0.172 \\
(0.251)\end{array}$ & $\begin{array}{c}-0.200 \\
(1.100)\end{array}$ \\
\hline $\begin{array}{l}\gamma_{\mathrm{i}, \mathrm{UK}} \\
(\mathrm{x} 100,000)\end{array}$ & $\begin{array}{l}-0.159 \\
(0.243)\end{array}$ & $\begin{array}{l}-0.018 \\
(0.256)\end{array}$ & $\begin{array}{c}0.437 \\
(0.664)\end{array}$ & $\begin{array}{l}-0.096 \\
(0.205)\end{array}$ & $\begin{array}{l}-0.099 \\
(0.053)\end{array}$ & $\begin{array}{c}0.835 \\
(2.070)\end{array}$ & $\begin{array}{l}-0.184 \\
(0.221)\end{array}$ & $\begin{array}{c}0.304 \\
(0.426)\end{array}$ & $\begin{array}{c}2.060 \\
(1.360)\end{array}$ \\
\hline $\begin{array}{l}\gamma_{\mathrm{i}, \text { Canada }} \\
(\mathrm{x} 100,000)\end{array}$ & $\begin{array}{c}0.132 \\
(0.268)\end{array}$ & $\begin{array}{c}-0.012 \\
(0.209)\end{array}$ & $\begin{array}{l}-0.224 \\
(0.457)\end{array}$ & $\begin{array}{c}0.207 \\
(0.171)\end{array}$ & $\begin{array}{c}0.047 \\
(0.146)\end{array}$ & $\begin{array}{c}-3.190 \\
(1.01 * * *)\end{array}$ & $\begin{array}{c}1.750 \\
\left(0.66^{* * *}\right)\end{array}$ & $\begin{array}{c}-0.044 \\
(0.420)\end{array}$ & $\begin{array}{l}-1.210 \\
(1.040)\end{array}$ \\
\hline $\begin{array}{l}\gamma_{\mathrm{i}, \text { Mexico }} \\
(\mathrm{x} 100,000)\end{array}$ & $\begin{array}{c}0.337 \\
(0.227)\end{array}$ & $\begin{array}{l}-0.052 \\
(0.166)\end{array}$ & $\begin{array}{l}-0.321 \\
(0.441)\end{array}$ & $\begin{array}{c}0.225 \\
(0.215)\end{array}$ & $\begin{array}{c}0.006 \\
(0.040)\end{array}$ & $\begin{array}{c}0.892 \\
(0.919)\end{array}$ & $\begin{array}{c}0.015 \\
(0.274)\end{array}$ & $\begin{array}{c}0.367 \\
(0.320)\end{array}$ & $\begin{array}{c}0.471 \\
(0.941)\end{array}$ \\
\hline ARCH-LM & 0.1899 & 0.3110 & 3.4280 & 0.0012 & 0.1338 & 0.5010 & 0.7592 & 0.5880 & 0.3348 \\
\hline
\end{tabular}

NOTES: The $\delta$ coefficients measure mean return transmissions; the $\gamma$ coefficients measure volatility transmissions.

** Significant at the $5 \%$ level. $\quad * * *$ Significant at the $1 \%$ level. 
Table 5 reports the results for currencies from emerging markets. Brazil and Mexico show strong price spillover effects. In this model Mexico exhibits significant results for every commodity except rice. The results for Mexico are even stronger here than in Table 4, adding significant results for hogs and natural gas to the previous significant relationships. It is possible that the absence of Canada as a control variable in this model is allowing for the additional significant results. Brazil has a significant relationship with six of the nine commodities and is uniformly significant across the grains complex. South Korea shows a much lower degree of interaction with significant results for only soybeans and crude oil.

Table 5. Mean and volatility spillovers from emerging economies

\begin{tabular}{|c|c|c|c|c|c|c|c|c|c|}
\hline & & & & & Feeder & & Live & & Natural \\
\hline & Corn & Rice & Soybeans & Wheat & Cattle & Hogs & Cattle & Crude & Gas \\
\hline$\delta_{i, 3}$ & $\begin{array}{c}2.658 \\
(1.754)\end{array}$ & $\begin{array}{c}-5.512 \\
(3.245)\end{array}$ & $\begin{array}{c}-7.775 \\
(2.31 * * *)\end{array}$ & $\begin{array}{l}-3.500 \\
(2.034)\end{array}$ & $\begin{array}{c}7.988 \\
(5.324)\end{array}$ & $\begin{array}{l}-3.956 \\
(2.591)\end{array}$ & $\begin{array}{c}-14.371 \\
(10.454)\end{array}$ & $\begin{array}{l}-1.068 \\
(1.919)\end{array}$ & $\begin{array}{l}-1.797 \\
(1.392)\end{array}$ \\
\hline $\begin{array}{l}\delta_{i, \text { Brazil }} \\
(\mathrm{x} 1,000)\end{array}$ & $\begin{array}{c}-1.326 \\
(0.35 * * *)\end{array}$ & $\begin{array}{c}-1.114 \\
(0.37 * * *)\end{array}$ & $\begin{array}{c}-1.793 \\
(0.31 * * *)\end{array}$ & $\begin{array}{c}-0.954 \\
(0.38 * *)\end{array}$ & $\begin{array}{c}0.087 \\
(0.187)\end{array}$ & $\begin{array}{c}-1.456 \\
(0.62 * *)\end{array}$ & $\begin{array}{l}-0.382 \\
(0.234)\end{array}$ & $\begin{array}{c}-1.758 \\
(0.49 * * *)\end{array}$ & $\begin{array}{l}-1.146 \\
(0.713)\end{array}$ \\
\hline $\begin{array}{l}\delta_{i, \text { Mexico }} \\
(\mathrm{x} 1,000)\end{array}$ & $\begin{array}{c}-1.161 \\
(0.34 * * *)\end{array}$ & $\begin{array}{c}-0.603 \\
(0.353)\end{array}$ & $\begin{array}{c}-1.127 \\
(0.33 * * *)\end{array}$ & $\begin{array}{c}-0.788 \\
(0.392 * *)\end{array}$ & $\begin{array}{c}-0.612 \\
\left(0.19^{* * *}\right)\end{array}$ & $\begin{array}{c}0.900 \\
\left(0.45^{* *}\right)\end{array}$ & $\begin{array}{c}-0.788 \\
(0.27 * * *)\end{array}$ & $\begin{array}{c}-2.712 \\
(0.47 * * *)\end{array}$ & $\begin{array}{c}-1.785 \\
(0.70 * *)\end{array}$ \\
\hline $\begin{array}{l}\delta_{i, \mathrm{~S} . \text { Korea }} \\
(\mathrm{x} 1,000)\end{array}$ & $\begin{array}{c}-0.388 \\
(0.340)\end{array}$ & $\begin{array}{c}-0.554 \\
(0.303)\end{array}$ & $\begin{array}{c}-1.282 \\
(0.30 * * *)\end{array}$ & $\begin{array}{l}-0.320 \\
(0.410)\end{array}$ & $\begin{array}{c}0.028 \\
(0.176)\end{array}$ & $\begin{array}{c}-0.353 \\
(0.448)\end{array}$ & $\begin{array}{l}-0.192 \\
(0.204)\end{array}$ & $\begin{array}{c}-1.631 \\
(0.41 * * *)\end{array}$ & $\begin{array}{l}-0.621 \\
(0.602)\end{array}$ \\
\hline$\gamma_{\mathrm{i}, 2}$ & $\begin{array}{c}0.050 \\
(0.01 * * *)\end{array}$ & $\begin{array}{c}0.023 \\
(0.009 * *)\end{array}$ & $\begin{array}{c}0.048 \\
(0.01 * * *)\end{array}$ & $\begin{array}{c}0.030 \\
(0.01 * * *)\end{array}$ & $\begin{array}{c}0.028 \\
\left(0.01^{* * *}\right)\end{array}$ & $\begin{array}{c}0.144 \\
(0.2 * * *)\end{array}$ & $\begin{array}{c}0.027 \\
(0.015)\end{array}$ & $\begin{array}{c}0.079 \\
(0.02 * * *)\end{array}$ & $\begin{array}{c}0.048 \\
(0.01 * * *)\end{array}$ \\
\hline$\gamma_{\mathrm{i}, 3}$ & $\begin{array}{c}0.945 \\
(0.01 * * *)\end{array}$ & $\begin{array}{c}0.968 \\
(0.01 * * *)\end{array}$ & $\begin{array}{c}0.940 \\
(0.01 * * *)\end{array}$ & $\begin{array}{c}0.962 \\
(0.01 * * *)\end{array}$ & $\begin{array}{c}0.961 \\
(0.01 * * *)\end{array}$ & $\begin{array}{c}0.762 \\
(0.01 * * *)\end{array}$ & $\begin{array}{c}0.869 \\
(0.06 * * *)\end{array}$ & $\begin{array}{c}0.873 \\
(0.02 * * *)\end{array}$ & $\begin{array}{c}0.939 \\
(0.01 * * *)\end{array}$ \\
\hline $\begin{array}{l}\gamma_{\mathrm{i}, \text { Brazil }} \\
(100,000)\end{array}$ & $\begin{array}{c}0.443 \\
(0.173 * *)\end{array}$ & $\begin{array}{c}0.248 \\
(0.146)\end{array}$ & $\begin{array}{c}0.080 \\
(0.123)\end{array}$ & $\begin{array}{c}-0.128 \\
(0.212)\end{array}$ & $\begin{array}{c}-0.065 \\
(0.036)\end{array}$ & $\begin{array}{c}-2.990 \\
(0.20 * * *)\end{array}$ & $\begin{array}{c}0.014 \\
(0.191)\end{array}$ & $\begin{array}{c}1.310 \\
(0.671)\end{array}$ & $\begin{array}{c}0.065 \\
(0.859)\end{array}$ \\
\hline $\begin{array}{l}\gamma_{\mathrm{i}, \text { Mexico }} \\
(\mathrm{x} 100,000)\end{array}$ & $\begin{array}{c}0.056 \\
(0.128)\end{array}$ & $\begin{array}{c}-0.184 \\
(0.130)\end{array}$ & $\begin{array}{c}0.082 \\
(0.093)\end{array}$ & $\begin{array}{c}0.282 \\
(0.208)\end{array}$ & $\begin{array}{c}-0.016 \\
(0.047)\end{array}$ & $\begin{array}{c}2.710 \\
(0.98 * * *)\end{array}$ & $\begin{array}{l}-0.128 \\
(0.267)\end{array}$ & $\begin{array}{c}0.543 \\
(0.448)\end{array}$ & $\begin{array}{c}0.307 \\
(0.578)\end{array}$ \\
\hline $\begin{array}{l}\gamma_{\mathrm{i}, \mathrm{S} . \text { Korea }} \\
(\mathrm{x} 100,000)\end{array}$ & $\begin{array}{c}0.186 \\
(0.171)\end{array}$ & $\begin{array}{c}0.285 \\
(0.14 * * *)\end{array}$ & $\begin{array}{l}-0.009 \\
(0.135)\end{array}$ & $\begin{array}{c}0.289 \\
(0.183)\end{array}$ & $\begin{array}{c}0.083 \\
(0.04 * *)\end{array}$ & $\begin{array}{c}0.761 \\
(0.964)\end{array}$ & $\begin{array}{c}0.086 \\
(0.125)\end{array}$ & $\begin{array}{c}0.563 \\
(0.563)\end{array}$ & $\begin{array}{c}0.225 \\
(0.623)\end{array}$ \\
\hline ARCH-LM & 0.4727 & 0.5660 & 0.2778 & 0.0503 & 0.4332 & 0.5138 & 0.0871 & 0.2433 & 0.2953 \\
\hline
\end{tabular}

NOTES: The $\delta$ coefficients measure mean return transmissions; the $\gamma$ coefficients measure volatility transmissions.

** Significant at the $5 \%$ level. $\quad * * *$ Significant at the $1 \%$ level.

As with the results for Table 4, there is little evidence of volatility spillover from the emerging markets currency returns as there are only four significant relationships across the entire sample, although once again the commodity hogs accounts for two of those results, receiving volatility spillovers from both Brazil and Mexico.

The results for the model using pacific rim currencies are shown in Table 6. Only Australia exhibits price spillover effects broadly across the commodities sample, showing uniformly significant results for both the grains and energy complexes, although it shows no significant results for commodities in the livestock complex. However, the $\delta$ coefficients are all positive, indicating that a positive innovation in the mean of the currency distribution (implying an appreciation of the US dollar vs. the Australian dollar) generates a positive innovation in the commodity returns distribution (implying an increase in commodity prices). Likewise, Japan shows significant price spillover results with only two commodities (feeder cattle and crude oil), but these are the same two commodities that Japan affected in the model shown in Table 4, and, as with the results for Australia, the $\delta$ coefficients are positive. New Zealand exhibits no significant results and South Korea, consistent with the model shown in Table 5, transmits mean returns to soybeans and crude oil. 
Table 6. Mean and volatility spillovers from pacific rim countries

\begin{tabular}{|c|c|c|c|c|c|c|c|c|c|}
\hline & & & & & Feeder & & Live & & Natural \\
\hline & Corn & Rice & Soybeans & Wheat & Cattle & Hogs & Cattle & Crude & Gas \\
\hline$\delta_{i, 3}$ & $\begin{array}{c}8.029 \\
(1.91 * * *)\end{array}$ & $\begin{array}{c}-6.370 \\
(3.03 * *)\end{array}$ & $\begin{array}{c}-2.602 \\
(2.123)\end{array}$ & $\begin{array}{l}-3.783 \\
(2.047)\end{array}$ & $\begin{array}{c}7.903 \\
(5.252)\end{array}$ & $\begin{array}{c}-15.86 \\
(6.83 * *)\end{array}$ & $\begin{array}{c}-15.68 \\
(12.665)\end{array}$ & $\begin{array}{c}0.821 \\
(1.982)\end{array}$ & $\begin{array}{l}-1.838 \\
(1.375)\end{array}$ \\
\hline $\begin{array}{l}\delta_{i, \text { Australia }} \\
(\mathrm{x} 1,000)\end{array}$ & $\begin{array}{c}1.456 \\
(0.53 * * *)\end{array}$ & $\begin{array}{c}1.746 \\
(0.54 * * *)\end{array}$ & $\begin{array}{c}3.385 \\
\left(0.45^{* * *}\right)\end{array}$ & $\begin{array}{c}1.894 \\
\left(0.61^{* * *}\right)\end{array}$ & $\begin{array}{c}0.387 \\
(0.297)\end{array}$ & $\begin{array}{c}1.029 \\
(0.644)\end{array}$ & $\begin{array}{c}0.715 \\
(0.392)\end{array}$ & $\begin{array}{c}6.261 \\
(0.71 * * *)\end{array}$ & $\begin{array}{c}2.886 \\
\left(1.1^{* * *}\right)\end{array}$ \\
\hline $\begin{array}{l}\delta_{i, \text { Japan }} \\
(\mathrm{x} 1,000)\end{array}$ & $\begin{array}{l}0.226 \\
(0.34)\end{array}$ & $\begin{array}{c}0.188 \\
(0.311)\end{array}$ & $\begin{array}{c}0.321 \\
(0.275)\end{array}$ & $\begin{array}{c}0.345 \\
(0.378)\end{array}$ & $\begin{array}{c}0.581 \\
\left(0.16^{* * *}\right)\end{array}$ & $\begin{array}{c}0.535 \\
(0.359)\end{array}$ & $\begin{array}{c}0.401 \\
(0.193)\end{array}$ & $\begin{array}{c}1.523 \\
\left(0.40^{* * *}\right)\end{array}$ & $\begin{array}{c}0.074 \\
(0.586)\end{array}$ \\
\hline $\begin{array}{l}\delta_{i, \text { New Zealand }} \\
(\mathrm{x} 1,000)\end{array}$ & $\begin{array}{c}0.544 \\
(0.536)\end{array}$ & $\begin{array}{c}0.349 \\
(0.534)\end{array}$ & $\begin{array}{l}-0.395 \\
(0.450)\end{array}$ & $\begin{array}{c}0.508 \\
(0.604)\end{array}$ & $\begin{array}{c}0.020 \\
(0.288)\end{array}$ & $\begin{array}{c}-0.708 \\
(0.629)\end{array}$ & $\begin{array}{c}0.181 \\
(0.368)\end{array}$ & $\begin{array}{l}-0.271 \\
(0.702)\end{array}$ & $\begin{array}{c}0.27 \\
(1.096)\end{array}$ \\
\hline $\begin{array}{l}\delta_{i, \mathrm{~S} . \text { Korea }} \\
(\mathrm{x} 1,000)\end{array}$ & $\begin{array}{c}-0.283 \\
(0.345)\end{array}$ & $\begin{array}{l}-0.356 \\
(0.308)\end{array}$ & $\begin{array}{c}-0.878 \\
(0.30 * * *)\end{array}$ & $\begin{array}{r}-0.0714 \\
(0.415)\end{array}$ & $\begin{array}{r}-0.0174 \\
(0.177)\end{array}$ & $\begin{array}{l}-0.143 \\
(0.400)\end{array}$ & $\begin{array}{c}-0.076 \\
(0.214)\end{array}$ & $\begin{array}{c}-0.848 \\
(0.404 * *)\end{array}$ & $\begin{array}{l}-0.109 \\
(0.591)\end{array}$ \\
\hline$\gamma_{\mathrm{i}, 2}$ & $\begin{array}{c}0.049 \\
(0.01 * * *)\end{array}$ & $\begin{array}{c}0.022 \\
(0.01 * * *)\end{array}$ & $\begin{array}{c}0.050 \\
(0.01 * * *)\end{array}$ & $\begin{array}{c}0.030 \\
(0.01 * * *)\end{array}$ & $\begin{array}{c}0.031 \\
(0.01 * * *)\end{array}$ & $\begin{array}{l}0.001 \\
(0.004\end{array}$ & $\begin{array}{c}0.029 \\
(0.032)\end{array}$ & $\begin{array}{c}0.054 \\
(0.012)\end{array}$ & $\begin{array}{c}0.049 \\
(0.01 * * *)\end{array}$ \\
\hline$\gamma_{i, 3}$ & $\begin{array}{c}0.938 \\
(0.01 * * *)\end{array}$ & $\begin{array}{c}0.970 \\
(0.01 * * *)\end{array}$ & $\begin{array}{c}0.940 \\
(0.01 * * *)\end{array}$ & $\begin{array}{c}0.962 \\
(0.01 * * *)\end{array}$ & $\begin{array}{c}0.957 \\
(0.01 * * *)\end{array}$ & $\begin{array}{c}0.895 \\
0.06 * * *)\end{array}$ & $\begin{array}{c}-0.243 \\
(0.226)\end{array}$ & $\begin{array}{c}0.919 \\
(0.015)\end{array}$ & $\begin{array}{c}0.938 \\
(0.01 * * *)\end{array}$ \\
\hline $\begin{array}{l}\gamma_{\mathrm{i}, \text { Australia }} \\
(100,000)\end{array}$ & $\begin{array}{c}-0.0534 \\
(0.208)\end{array}$ & $\begin{array}{l}0.0227 \\
(0.139)\end{array}$ & $\begin{array}{c}0.370 \\
(0.511)\end{array}$ & $\begin{array}{c}-0.228 \\
(0.329)\end{array}$ & $\begin{array}{c}-0.157 \\
(0.04 * * *)\end{array}$ & $\begin{array}{c}1.060 \\
(0.314)\end{array}$ & $\begin{array}{c}1.020 \\
(0.539)\end{array}$ & $\begin{array}{c}0.693 \\
(0.563)\end{array}$ & $\begin{array}{c}0.764 \\
(1.440)\end{array}$ \\
\hline $\begin{array}{l}\gamma_{\mathrm{i}, \mathrm{Japan}} \\
(\mathrm{x} 100,000)\end{array}$ & $\begin{array}{l}0.135 \\
(119)\end{array}$ & $\begin{array}{c}0.267 \\
\left(0.10^{* * *}\right)\end{array}$ & $\begin{array}{c}0.0424 \\
(0.182)\end{array}$ & $\begin{array}{r}-0.0725 \\
(0.102)\end{array}$ & $\begin{array}{r}-0.0508 \\
(0.039)\end{array}$ & $\begin{array}{c}-0.235 \\
(0.03 * * *)\end{array}$ & $\begin{array}{c}-0.283 \\
(0.215)\end{array}$ & $\begin{array}{c}-0.274 \\
(0.251)\end{array}$ & $\begin{array}{c}0.058 \\
(0.575)\end{array}$ \\
\hline $\begin{array}{l}\gamma_{i, \text { New Zealand }} \\
(\mathrm{x} 100,000)\end{array}$ & $\begin{array}{c}0.497 \\
(0.303)\end{array}$ & $\begin{array}{c}-0.318 \\
\left(0.15^{* *}\right)\end{array}$ & $\begin{array}{c}0.0325 \\
(0.306)\end{array}$ & $\begin{array}{c}0.553 \\
(0.346)\end{array}$ & $\begin{array}{c}0.156 \\
(0.04 * * *)\end{array}$ & $\begin{array}{l}-1.650 \\
(0.316)\end{array}$ & $\begin{array}{c}0.011 \\
(0.396)\end{array}$ & $\begin{array}{c}0.630 \\
(0.621)\end{array}$ & $\begin{array}{l}-1.470 \\
(1.210)\end{array}$ \\
\hline 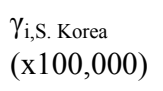 & $\begin{array}{c}0.175 \\
(0.192)\end{array}$ & $\begin{array}{c}0.216 \\
(0.130)\end{array}$ & $\begin{array}{r}-0.0695 \\
(0.176)\end{array}$ & $\begin{array}{c}0.298 \\
(0.168)\end{array}$ & $\begin{array}{c}0.0951 \\
(0.038 * *)\end{array}$ & $\begin{array}{c}-0.017 \\
(0.234)\end{array}$ & $\begin{array}{c}-0.076 \\
(0.284)\end{array}$ & $\begin{array}{c}0.297 \\
(0.240)\end{array}$ & $\begin{array}{c}0.421 \\
(0.619)\end{array}$ \\
\hline ARCH-LM & 0.5068 & 0.7532 & 0.4206 & 0.0237 & 0.2274 & 0.0488 & 0.0576 & 0.0264 & 0.2485 \\
\hline
\end{tabular}

NOTES: The $\delta$ coefficients measure mean return transmissions; the $\gamma$ coefficients measure volatility transmissions.

$* *$ Significant at the $5 \%$ level. ***Significant at the $1 \%$ level.

Consistent with previous results, the presence of volatility transmissions from pacific rim countries is sporadic. Japan once again transmits volatility spillovers to hogs, and feeder cattle receives transmissions from three of the four currencies (Australia, New Zealand, and South Korea). Rice receives volatility spillover from two currencies (Japan and New Zealand) although South Korea does not repeat the significant results shown in Table 5. We note that neither of the energy commodities (crude oil and natural gas) receives volatility transmissions from any currency in any of the models.

Overall these results document that price spillover effects are common and that volatility spillover effects are less pronounced and tend to occur for lower volume commodities. It is noted that this study is limited by the inability of the model to account for volume or other external factors (such as contango or backwardation in the futures curve for each commodity) driving the spillover effects. Future studies in this area could apply foreign exchange-to-commodity spillover effects to hedging models such as the minimum variance hedge ratio examined by Lien and Yang (2008) and asset pricing models such as the Kalman filter model used by Cortazar and Severino (2008).

\section{Conclusion}

Previous studies of commodity pricing effects have documented the need to account for volatility and other information spillover effects between securities. Consistent with these observations, our tests show broad based mean-return transmissions from certain currencies to commodities traded within the CME Group. In particular, price spillover effects are broadly distributed across the commodities sample by mean-return innovations in exchange rates for the currencies of the EU, Canada, Mexico, Brazil, and Australia. Each of these countries are either large GDP economies, major US trade partners, and/or major exporters of agricultural commodities. We find less evidence of 
volatility spillover effects, although the volatility transmissions observed tend to happen for the lower liquidity commodities such as hogs, feeder cattle, and rice. These results are consistent with contagion theory as proposed by King and Wadhwani (1990).

The results are important as they demonstrate the need to account for price and volatility spillover effects from foreign exchange rates to commodities when considering asset pricing and price discovery dynamics for commodities, and also when creating commodity based hedging models.

\section{References}

Badshah, I. U., Frijns, B., \& Tourani-Rad, A. (2013). Contemporaneous spill-over among equity, gold, and exchange rate implied volatility indices. Journal of Futures Markets, 33, 555-572. http://dx.doi.org/10.1002/fut.21600

Black, F. (1976). Studies of stock market volatility changes. Proceedings of the American Statistical Association, Business and Economics Studies Section, pp. 177-181.

Bollerslev, T., \& Wooldridge, J.M. (1992). Quasi-maximum likelihood estimation and inference in dynamic models with time varying covariances. Econometric Reviews, 11, 143-172. http://dx.doi.org/10.1080/07474939208800229

Chng, M. T. (2009). Economic linkages across commodity futures: Hedging and trading implications. Journal of Banking and Finance, 33, 958-970. http://dx.doi.org/10.1016/j.jbankfin.2008.10.006

Conrad, J., Gultekin, M., \& Kaul, G. (1991). Asymmetric predictability of conditional variances. Review of Financial Studies, 4, 597-622. http://dx.doi.org/10.1093/rfs/4.4.597

Cortazar, G., Milla, C., \& Severino, F. (2008). A multicommodity model of futures prices: Using futures prices of one commodity to estimate the stochastic process of another. Journal of Futures Markets, 28, 537-560. http://dx.doi.org/10.1002/fut.20322

Driesprong, G., Jacobsen, B., \& Maat, B. (2008). Striking oil: Another puzzle? Journal of Financial Economics, 89, 307-327. http://dx.doi.org/10.1016/j.jfineco.2007.07.008

Duan, J-C., \& Pliska, S. R. (2004). Option valuation with cointegrated asset prices. Journal of Economic Dynamics \& Control, 28, 727-754. http://dx.doi.org/10.1016/S0165-1889(03)00042-3

Hamao, Y., Masulis, R., \& Ng, V. (1990). Correlation in price changes and volatility across international stock markets. Review of Financial Studies, 3, 281-308. http://dx.doi.org/10.1093/rfs/3.2.281

Kanas, A. (1998). Volatility spillovers across equity markets: European evidence. Applied Financial Economics, 8 , 245-256. http://dx.doi.org/10.1080/096031098333005

King, M. A., \& Wadhwani, S. (1990). Transmission of volatility between stock markets. The Review of Financial Studies, 3, 5-13. http://dx.doi.org/10.1093/rfs/3.1.5

Koutmos, G., \& Booth, G.G. (1995). Asymmetric volatility transmission in international stock markets. Journal of International Money and Finance, 14, 747-762. http://dx.doi.org/10.1016/0261-5606(95)00031-3

Lien, D., \& Yang, L. (2008). Asymmetric effect of basis on dynamic futures hedging: Empirical evidence from commodity markets. Journal of Banking and Finance, 32, 187-198. http://dx.doi.org/10.1016/j.jbankfin.2007.01.026

Liu, Y. A., \& Pan, M-S. (1997). Mean and volatility spillover effects in the U.S. and Pacific-Basin stock markets. Multinational Finance Journal, 1, 47-62.

Nakajima, K., \& Ohashi, K. (2012). A Cointegrated Commodity Pricing Model. Journal of Futures Markets, 32, 995-1033. http://dx.doi.org/10.1002/fut.20553

Theodossiou, P., Kahya, E., Koutmos, G., \& Christofi, A. (1997). Volatility reversion and correlation structure of returns in major international stock markets. The Financial Review, 32, 205-224. http://dx.doi.org/10.1111/j.1540-6288.1997.tb00422.x

Theodossiou, P., \& Lee, U. (1993). Mean and volatility spillovers across major national stock markets: Further evidence. The Journal of Financial Research, 16, 337-350.

Trolle, A. B., \& Schwartz, E. S. (2009). Unspanned stochastic volatility and the pricing of commodity derivatives. Review of Financial Studies, 22, 4423-4461. http://dx.doi.org/10.1093/rfs/hhp036 
Tse, Y. (1998). International transmission of information: Evidence from the Euroyen and Eurodollar futures markets. Journal of International Money and Finance, 17, 909-929. http://dx.doi.org/10.1016/S0261-5606(98)00034-5

\section{Notes}

Note 1. Indirect quotes are used for the exchange rate data for each series (i.e., units of foreign currency per \$1 US). An increase the currency rate represents an appreciation of the US dollar making US exports more expensive and imports to the US from other countries less expensive.

Note 2. Some overlap appears across the different groups. Most notably Japan, Mexico, and South Korea appear in more than one group. This allows us to observe simultaneous spillover effects from multiple countries within three different groups of interest (i.e., large GDP and US trade partners, emerging economies, and pacific rim countries).

Note 3. The World Bank reports the European Union, Japan, and the United Kingdom as the three largest GDP countries excluding the US and China (http://data.worldbank.org/data-catalog/GDP-ranking-table), and the International Trade Commission ranks the EU, Canada, Mexico, and Japan as the four largest US trading partners excluding China (http://dataweb.usitc.gov).

Note 4. Brazil is the 3rd largest agricultural exporter in the world with $\$ 6.2 \mathrm{~B}$ of agricultural trade per year with the US. Brazil exports beef and pork to the US and imports wheat from the US (http://www.fas.usda.gov/country/Brazil/Brazil.asp). Mexico is the second largest agricultural trade partner for the US, accounting for $11 \%$ of US ag-imports and $13 \%$ of US ag-exports. Approximately $80 \%$ of Mexico's ag-exports go to the US. (http://mexico.usembassy.gov/eng/eataglance trade.html). South Korea is the fifth largest market for US ag-exports and the US is the primary source of South Korea's ag-imports (http://seoul.usembassy.gov/fcs_exporting.html).

Note 5. Australia and New Zealand are major agricultural economies within Asia, although they have lower rankings as US agricultural trade partners. As a trade partner with the US, Australia is the 20th largest ag-export market and 8th largest ag-import market (http://www.ustr.gov/countries-regions/southeast-asia-pacific/australia). Likewise, New Zealand's trade relationship with the US ranks it as the 13th largest ag-import market (http://www.ustr.gov/countries-regions/southeast-asia-pacific/new-zealand). 